International Journal of Pure and Applied Mathematics

Volume 107 No. 3 2016, 709-721

ISSN: 1311-8080 (printed version); ISSN: 1314-3395 (on-line version)

url: http://www.ijpam.eu

doi: 10.12732/ijpam.v107i3.19

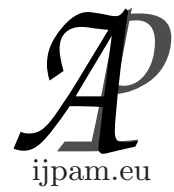

\title{
BOUNDARY DETECTION ALGORITHM BASED ON SEMI-SUBCOMPLEXES
}

\author{
G. Sai Sundara Krishnan ${ }^{1}$, N. Vijaya ${ }^{2} \S$ \\ ${ }^{1}$ PSG College of Technology \\ Coimbatore, INDIA \\ ${ }^{2}$ Adhiyamaan College of Engineering \\ Hosur, INDIA
}

\begin{abstract}
In this paper, the concept of semi-open subcomplexes in abstract cellular complex is introduced and some of their properties are investigated by describing the notions of semifrontier, semi-interior and semi-closure. An efficient algorithm for tracing the semi-frontier of an image is introduced and is implemented through MATLAB. Finally, the semi-frontier algorithm is compared with the boundary tracing algorithm [G. Sai Sundara Krishnan, 2012].
\end{abstract}

AMS Subject Classification: 57Q05, 68U10

Key Words: semi-open subcomplex, semi-closed subcomplex, semi-frontier, semi-interior, semi-closure, semi-frontier tracing algorithm, boundary tracing

\section{Introduction}

Digital topology is the study of topological properties on digital images and it plays a very important role in digital image processing. Rosenfeld [1] represented a digital image by a graph whose nodes are pixels and whose edges are linking adjacent pixels to each other. He named the resultant graph the neighborhood graph. But this representation contains two paradoxes namely connectivity and boundary paradoxes. Kovalevsky [8] introduced the notion of abstract cellular complexes to study the structure of digital images and introduced axiomatic digital topology [12] which has no paradoxes. Moreover he showed that every finite topological space with separation property is isomor-

Received: November 14, 2015

Published: April 21, 2016

§ Correspondence author (c) 2016 Academic Publications, Ltd.

url: www.acadpubl.eu 
phic to an abstract cellular complex. Sang-Eoc Han [18] introduced the notions of continuity and homeomorphism between axiomatic locally finite spaces. In this paper, the concept of semi-open subcomplex in abstract cellular complex is introduced and some of their basic properties are studied which enable us to study the structure of digital images through semi-open subcomplexes. Further, the relationship between a semi-open subcomplex and a homogeneously n-dimensional subcomplex are studied and the notions of quasi-solid, semiregion are introduced. Further, a new algorithm for tracing the semi-frontier of an image using Kovalevsky's chain code is introduced. Finally, a comparative study between the semi-frontier tracing algorithm and boundary tracing [17] algorithm on various images is presented.

\section{Basic Notions}

In this section some basic definitions are recalled.

Definition 2.1. An abstract cellular complex $(A C C) C=(E, B$, dim $)$ is a set $E$ of abstract elements provided with an antisymmetric, irreflexive, and transitive binary relation $B \subset E \times E$ called the bounding relation, and with a dimension function $\operatorname{dim}: E \rightarrow I$ from $E$ into the set $I$ of non-negative integers such that $\operatorname{dim}\left(e^{\prime}\right)<\operatorname{dim}\left(e^{\prime \prime}\right)$ for all pairs $\left(e^{\prime}, e^{\prime \prime}\right) \in B$.

Definition 2.2. A subcomplex $S=\left(E^{\prime}, B^{\prime}\right)$ of a given K-complex $C=$ $(E, B)$ is a $\mathrm{K}$-complex whose set $E^{\prime}$ is the subset of $E$ and the relation $B^{\prime}$ is an intersection of $B$ with $E^{\prime} \times E^{\prime}$.

Definition 2.3. A subcomplex $S$ of $C$ is called open in $C$ if for every element $e^{\prime}$ of $S$ all elements of $C$ which are bounded by $e^{\prime}$ are also in $S$.

Definition 2.4. The smallest subset of a set $S$ which contains a given cell $c \in S$ and is open in $S$ is called smallest neighborhood of $c$ relative to $S$ and is denoted by $S O N(c, S)$.

Definition 2.5. The smallest subset of a set $S$ which contains a given cell $c \in S$ and is closed in $S$ is called the closure of $c$ relative to $S$ and denoted by $C l(c, S)$.

Definition 2.6. The frontier of a subcomplex $S$ of an abstract cellular complex $C$ relative to $C$ is the subcomplex $\operatorname{Fr}(S, C)$ containing of all cells $c$ of $C$ such that the $S O N(c)$ contains cells both of $S$ and of its complement $C-S$.

Definition 2.7. Let $t$ and $T$ be subsets of the space $S$ such that $t \subseteq T \subseteq S$. The set $t-\operatorname{Fr}(t, T)$ is called the interior of $t$ in $T$ and it is denoted by $\operatorname{Int}(t, T)$. 
Definition 2.8. An n-dimensional complex $C^{n}$ is called homogeneously n-dimensional when each of its cells of dimension less than $n$ bounds at least one n-cell of $C^{n}$.

Definition 2.9. A subcomplex $S^{n}$ of an n-dimensional complex $C^{n}$ is called solid iff it is homogeneously n-dimensional and contains the subcomplex $\operatorname{Int}\left(C l\left(S^{n}, C^{n}\right), C^{n}\right)$.

\section{Semi-Open Subcomplexes in Abstract Cellular Complex}

Definition 3.1. A subcomplex $S$ in an abstract cellular complex $C$ is called semi-open subcomplex if there exists an open subcomplex $O$ such that $O \subseteq S \subseteq C l(O)$ where $C l$ denotes the closure operator in $C$.

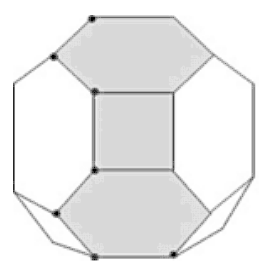

Figure 3.1: Examples of semi-open subcomplexes (surface of a polyhedron) in $2 \mathrm{D}$

Remark 3.1. It follows from the Definition 3.1 that an example of a semi-open subcomplex can be a pixel with at least one element of its frontier.

Theorem 3.1. A subcomplex $S$ of an abstract cellular complex $C$ is semi-open subcomplex if and only if $S \subseteq C l(\operatorname{Int}(S))$

Proof. Suppose $S \not \subset C l(\operatorname{Int}(S))$, then there exist a cell $x \in S$ such that $x \notin C l(\operatorname{Int}(S))$. This implies that $x \notin \operatorname{Int}(S)$ and $x \notin \operatorname{Fr}((\operatorname{Int}(S))) . x \notin \operatorname{Int}(S)$ implies that there exist no open subcomplex $O$ containing $x$ such that $O \subseteq S$ and $x \notin \operatorname{Fr}(\operatorname{Int}(S))$ implies that there exists no open subcomplex $O$ contained in $S$ such that $x \in \operatorname{Fr}((O))$. Hence there exist no open subcomplex $O$ such that $O \subseteq S \subseteq \operatorname{Int}(O) \cup \operatorname{Fr}(O)=C l(O))$. This is a contradiction to the assumption that $S$ is a semi-open subcomplex in $C$. Converse part is obvious from the definition of $\operatorname{Int}(S)$ while $\operatorname{Int}(S)$ is an open subcomplex contained in $S$.

Theorem 3.2. Every open subcomplex is a semi-open subcomplex.

Proof. Proof is follows directly from Theorem 3.1 
Remark 3.2. The converse of above Theorem 3.2 need not be true.
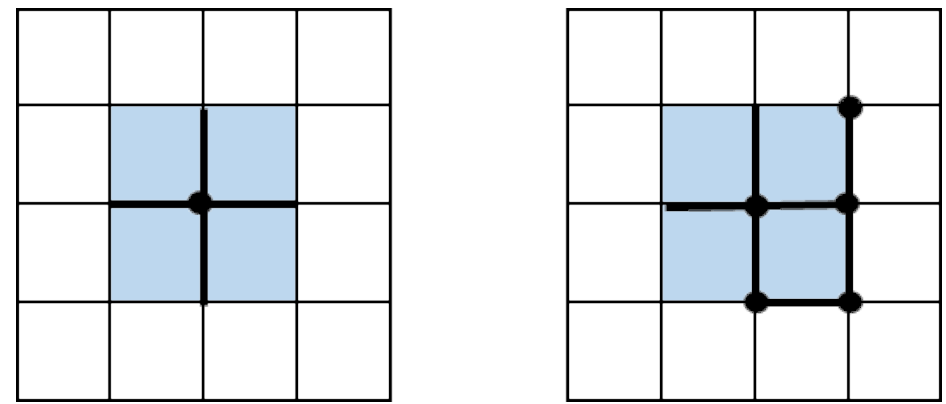

Figure 3.2: Example of open and semi-open subcomplexes

Lemma 3.1. If $S$ is a subcomplex of an abstract cellular complex $C$ then the following equations hold:

(i) $C-\operatorname{Int}(S)=C l(C-S)$

(ii) $C-C l(S)=\operatorname{Int}(C-S)$

Definition 3.2. A subcomplex $S$ of an abstract cellular complex $C$ is called semi-closed if $C-S$ is semi-open.

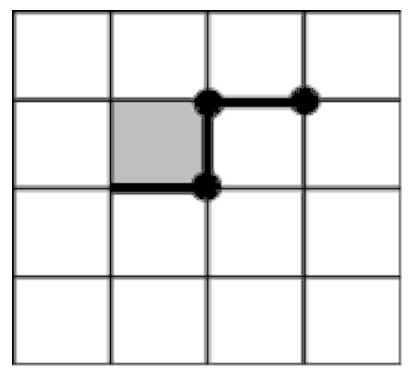

Figure 3.3: Example for semi-closed subcomplex.

Theorem 3.3. A subcomplex $S$ of an abstract cellular complex $C$ is semi-closed if and only if $\operatorname{Int}(C l(S)) \subseteq S$.

Proof. Proof is follows directly from Theorem 3.1 and Lemma 3.1.

Lemma 3.2. If $S_{1}$ and $S_{2}$ are any two subcomplexes of an abstract cellular complex $C$ and if $S_{1} \subseteq S_{2}$, then

(i) $\operatorname{Int}\left(S_{1}\right) \subseteq \operatorname{Int}\left(S_{2}\right)$ 
(ii) $\operatorname{Fr}\left(S_{1}\right) \subseteq C l\left(S_{2}\right)$

(iii) $C l\left(S_{1}\right) \subseteq C l\left(S_{2}\right)$

Proof. Proof (i) is follows directly from the Definition 2.7

(ii) Let $x \in \operatorname{Fr}\left(S_{1}\right)$. This implies that $S O N(x)$ intersects with both $S_{1}$ and $C-S_{1}$. If $S O N(x) \subseteq S_{2}$, then $x \in \operatorname{Int}\left(S_{2}\right)$ and if $S O N(x) \not \subset S_{2}$, then $x \in$ $\operatorname{Fr}\left(S_{2}\right)$. Hence $x \in \operatorname{Int}\left(S_{2}\right) \cup F\left(S_{2}\right)=C l\left(S_{2}\right)$.

(iii) Proof is follows directly from (i) and (ii)

Theorem 3.4. If $S_{1}$ and $S_{2}$ are two semi-open subcomplexes of an abstract cellular complex $C$, then $S_{1} \cup S_{2}$ is also semi-open subcomplex.

Proof. Given $S_{1}$ and $S_{2}$ are two semi-open subcomplexes of $C$. This implies that $S_{1} \subseteq C l\left(\operatorname{Int}\left(S_{1}\right)\right)$ and $S_{2} \subseteq C l\left(\operatorname{Int}\left(S_{2}\right)\right)$. This implies that $S_{1} \cup S_{2} \subseteq$ $C l\left(\operatorname{Int}\left(S_{1}\right)\right) \cup \subseteq C l\left(\operatorname{Int}\left(S_{2}\right)\right) \subseteq C l\left(\operatorname{Int}\left(S_{1} \cup S_{2}\right)\right)$. Hence $S_{1} \cup S_{2}$ is a semi-open subcomplex in $\mathrm{C}$.

Remark 3.3. The intersection of any two semi-open subcomplexes need not be a semi-open subcomplex.

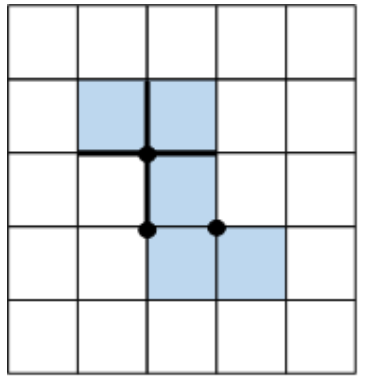

(a)

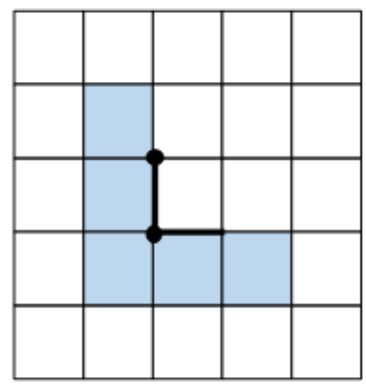

(b)

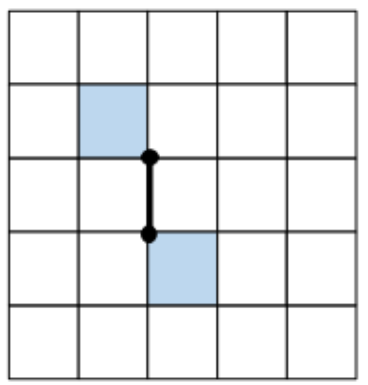

(c)

Figure 3.4: Example of semi-open subcomplexes(a) and (b); (c) intersection of (a) and (b) which is not semi-open

Theorem 3.5. Let $S_{1}$ be a semi-open subcomplex of an abstract cellular complex $C$ and $S_{1} \subseteq S_{2} \subseteq C l\left(S_{1}\right)$. Then $S_{2}$ is semi-open.

Proof. Given $S_{1}$ is semi-open. This implies that there exist an open subcomplex $O$ such that $O \subseteq S_{1} \subseteq C l(O)$. Hence $O \subseteq S_{2}$. By the Lemma3.2 (iii), we get $C l\left(S_{1}\right) \subseteq C l(O)$. Therefore $S_{2}$ is also semi-open. 
Theorem 3.6. If $S$ is homogeneously n-dimensional subcomplex of an n-dimensional complex $C$, then $\operatorname{Fr}(S)=\operatorname{Fr}(\operatorname{int}(S))$.

Proof. Suppose $\operatorname{Fr}(S) \neq \operatorname{Fr}(\operatorname{int}(S))$, implies that there is at least one lower dimensional cell $k$ of $\operatorname{Fr}(S)$ does not belong to $\operatorname{Fr}(\operatorname{Int}(S))$. This implies that the cell $k$ does not bound any n-cell of $S$. This contradicts the fact that $S$ is homogeneously n-dimensional.

Theorem 3.7. If $S$ is homogeneously n-dimensional subcomplex of an n-dimensional complex $C$, then it is semi-open.

Proof. Given $S$ is homogeneously n-dimensional subcomplex. By Definition of interior, all the principal cells of $S$ belongs to $\operatorname{Int}(S)$. Suppose $S \not \subset$ $C l(\operatorname{Int}(S))$, then there exist a lower dimensional cell $c \in S$ such that $c \notin$ $C l(\operatorname{Int}(S))$. This implies that the cell $c$ does not bound any n-cell of $\operatorname{Int}(S)$. This contradicts the fact that $S$ is homogeneously n-dimensional.

Theorem 3.8. If $S$ is strongly connected homogeneously n-dimensional subcomplex of an n-dimensional complex $C$, then it is semi-open.

Proof. Proof is follows directly from Theorem 3.5 and the Definition of semi-open.

Theorem 3.9. If a subcomplex $S$ of an n-dimensional complex $C$ is solid, then it semi-open.

Proof. If a subcomplex $S$ is solid, then it is homogeneously n-dimensional and contains the subcomplex $\operatorname{Int}(C l(S))$. This implies that $S \subseteq C l(\operatorname{Int}(S))$. Hence by Theorem 3.1, $S$ is semi-open.

Definition 3.3. Let $S$ be a non-empty subcomplex of an abstract cellular complex $C$. Then the semi-frontier of $S$ is the set of all elements $k$ of $C-S$, such that each neighborhood of $k$ contains elements of both $S$ and its complement $C-S$. It is denoted by $S F r$. 

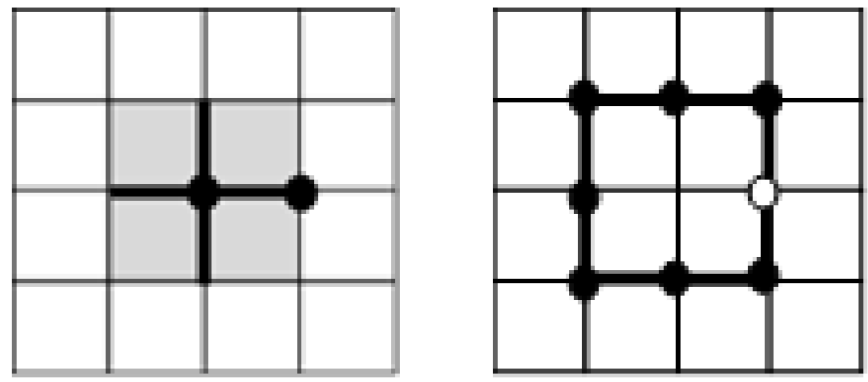

Figure 3.5: Example of semi-open subcomplex and its semi-frontier (From left to right).

Lemma 3.3. If $S$ is a subcomplex of an abstract cellular complex $C$, then $\operatorname{SFr}(S) \subseteq \operatorname{Fr}(S)$.

Lemma 3.4. If $S$ is a subcomplex of an abstract cellular complex $C$, then $\operatorname{SFr}(S) \cup S F r(C-S)=\operatorname{Fr}(S)$

Proof. Proof is follows directly from the Definition of $\mathrm{Fr}$ and $S F r$.

Definition 3.4. Let $S$ be a subcomplex of an abstract cellular complex $C$. The subcomplex $S-S F r(S)$ is called the semi-interior of $S$ and it is denoted by SInt.

Lemma 3.5. If $S$ is a subcomplex of an abstract cellular complex $C$, then $\operatorname{Int}(S) \subseteq \operatorname{SInt}(S)$.

Lemma 3.6. If $S$ is a semi-open subcomplex of an abstract cellular complex $C$, then $\operatorname{SInt}(S)$ is semi-open.

Theorem 3.10. Let $S$ be the subcomplex of an abstract cellular complex $C$ if and only if $\operatorname{SInt}(S)=S$.

Proof. Proof is follows directly from the definition of semi-interior.

Definition 3.5. Let $S$ be a subcomplex of an abstract cellular complex $C$. The subcomplex $S \cup \operatorname{SFr}(S)$ is called the semi-closure of $S$. It is denoted by $S C l$.

Definition 3.6. A subcomplex $S$ of an abstract cellular complex $C$ is called semi-open and connected if $S$ is connected and semi-open. 
Remark 3.4. If a subcomplex $S$ of an abstract cellular complex $C$ is open and connected, then it is semi-open and connected. But the converse is not true.

Definition 3.7. A subcomplex $S$ of an abstract cellular complex $C$ is called semi-region if $S$ semi-open, connected and solid.

Theorem 3.11. Every semi-open connected subcomplex $S$ of an n-dimensional complex $C$ is homogeneously n-dimensional.

Proof. Since $S$ is semi-open and connected, it contains no $k$-cell with $k<n$ which is not incident with an n-cell of $S$. Thus every $k$-cell with $k<n$ of $S$ is incident with an n-cell of $S$. Hence $S$ is homogeneously n-dimensional.

Definition 3.8. A subcomplex $S$ of an n-dimensional complex $C$ is called quasi-solid if and only if it is homogeneously n-dimensional and is contained in $C l(\operatorname{Int}(S, C), C)$.

Theorem 3.12. If a subcomplex $S^{n}$ of an n-dimensional complex is quasisolid then it is semi-open.

Proof. Proof is follows directly from the definition of quasi-solid and semiopen.

Theorem 3.13. Every solid subcomplex is quasi-solid.

Proof. Let $S$ be a solid subcomplex of an abstract cellular complex $C$. According to the Definition of a solid subcomplex, $S$ is homogeneously ndimensional and contains the subcomplex $\operatorname{Int}(C l(S, C), C)$. We get by Theorem $3.9, S$ is semi-open. Thus, $S$ is homogeneously n-dimensional and contained in $C l(\operatorname{Int}(S, C), C)$. Hence, by Definition of $3.17 S$ is quasi-solid.

Remark 3.5. From Theorems 3.7, 3.9, 3.13 and from Definition 2.8, 2.9 we have

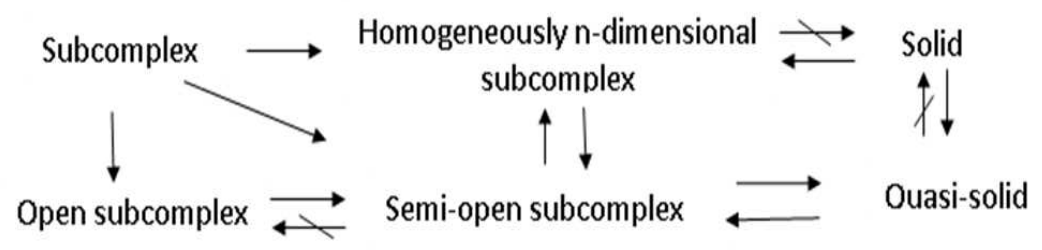




\section{Algorithm on Tracing the Semi-Frontier of an Image}

The basic idea of the proposed algorithm is to trace the semi-frontier of an image by considering the digitalized image as a semi-open subcomplexes in abstract cellular complex. The proposed algorithm is more efficient for the image which contains less number of components. The proposed algorithm consists of two major steps:

Step1: Before starting the tracing, the membership of the lower dimension cells (semi-open subcomplex) must be defined by the user whether it belongs to foreground or background of the image. The user can make the decision on the ground of some knowledge about the image.

Step2: Then, the image must be scanned row by row to find the starting point of each component. After finding the starting point, make the step along the boundary crack to the next boundary point using Kovalevsky's chain code.

The crack and end point of the crack both belongs to semi-frontier if it does not belong to foreground. The process stops when the starting point is reached again.

During each pass, the already visited cracks must be labeled to avoid multiple tracing.

\subsection{Algorithm}

The formal description of the algorithm is as follows:

Input: Given a digital pattern as a two dimensional abstract cellular complex Image containing points, cracks and pixels.

Output: A sequence SF of semi-frontier cracks and points.

Let $\mathbf{p}$ denote the current semi-frontier point.

Let $\mathbf{c}$ denote the current semi-frontier crack.

Begin

- Set Label to be empty

- Set $\mathbf{S F}$ to be empty

- Scan Image row by row until two subsequent pixels of different colors are found

- Set the upper end point of the crack $\mathbf{c}$ lying between the pixels of different colors as starting point $\mathbf{s}$ 
- Insert $\mathbf{s}, \mathbf{c}$ in $\mathbf{S F}$ if it does not belongs to foreground of the image

- Fix the direction as 1

- Move to next boundary point $\mathbf{p}$ along boundary crack $\mathbf{c}$

- do

- Insert p, c in SF if it does not belongs to foreground of the image.

- To recognize the next boundary crack test left and right pixels lying ahead of actual crack

- If Image[left] is foreground Change the direction in to (direction+1) $\% 4$

- else if Image[right] is background Change the direction in to $($ direction +3$) \% 4$

- Insert c in Label, if the direction is 1.

- Move to the next boundary point $\mathbf{p}$.

End while if $\mathbf{p}$ equal to $\mathbf{s}$

End

\section{Comparison with Boundary Tracing Algorithm}

In this section, Semi-Frontier tracing algorithm is compared with Boundary tracing algorithm [17] on the basis of various important factors. The various important factors of the comparison are as follows:

- Complexity

- Memory

\subsection{Experimental Results and Discussion}

Boundary tracing algorithm [17] traces the boundary of an image and semifrontier tracing algorithm [19] extracts the semi-frontier of an image. The semi-frontier elements are generally a small subset of the total number of elements that represent a boundary. Therefore, the allocation of memory space is highly reduced. Also, the amount of computation is reduced when the images 
are processed by means of certain semi-frontier features. Moreover, the time complexity of the both algorithms is $O\left(n^{4}\right)$. The result of this comparison on various images is shown below.
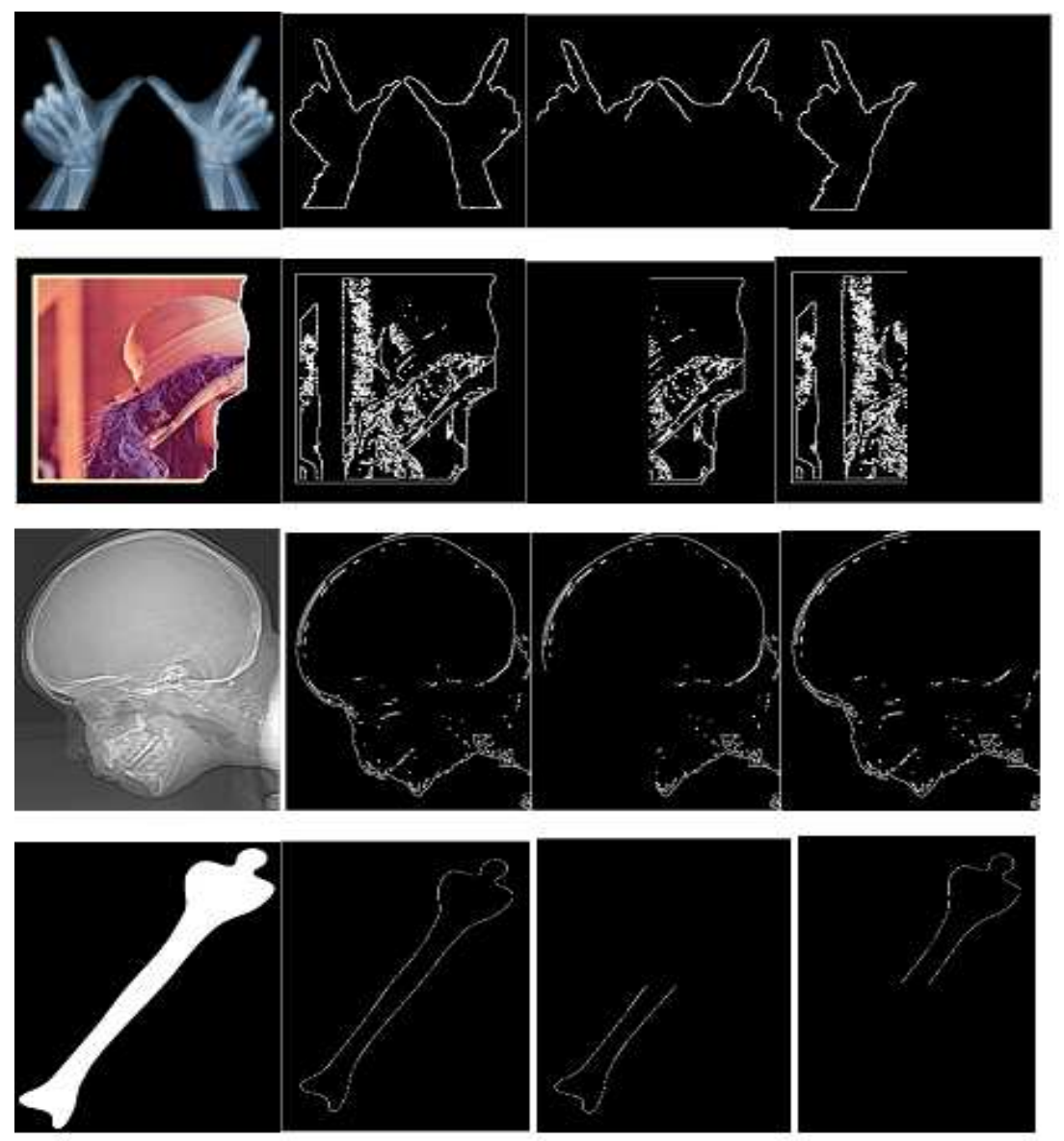

Figure 5.1: From left to right: Original image, boundary of an original image (second column) and semi-frontier of an original image (third and fourth column).

The detailed information of our experimental results on various images is illustrated in Figure 5.2. 


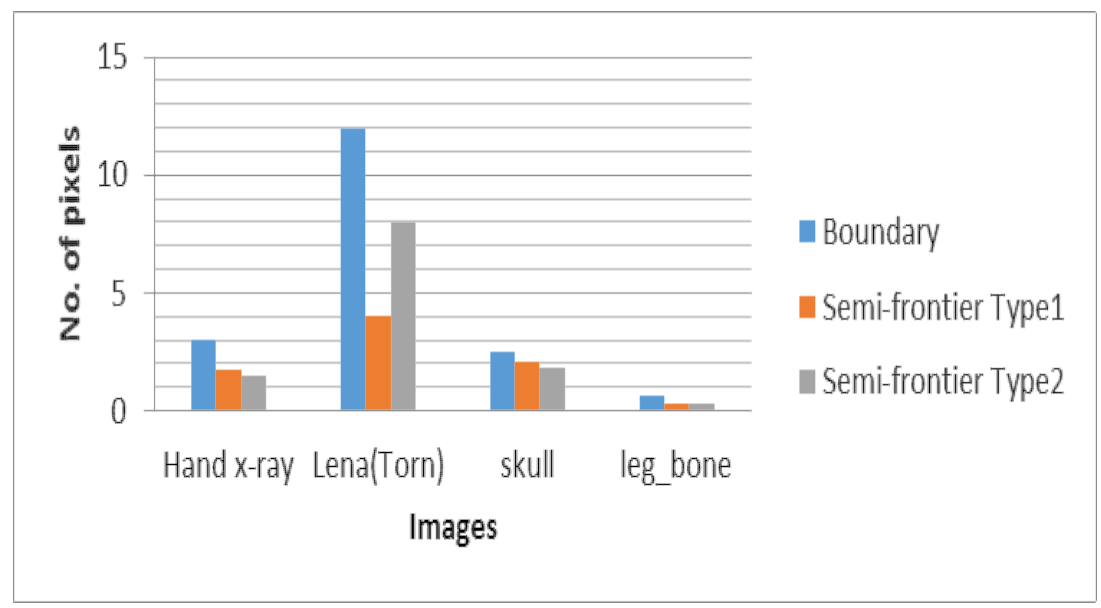

Figure 5.2. Comparative analysis on various images (all numbers expressed as percentages)

\subsection{Conclusion}

The comparison of the proposed algorithm with Boundary tracing algorithm have been discussed and also analyzed. The experimental result indicates that the Semi-Frontier tracing algorithm is computationally exponential both in memory and time.

\section{References}

[1] AzrielRosenfled, Digital toplogy, The American Mathematical Monthly, 8(1979), 621-630.

[2] N.Biswas, on some mapping in topological spaces, Bull. Cal. Math. Soc., 61(1969),127135.

[3] S.G.crossley and S.K.Hildebrand, semi-topological properties, Fund. Math., 74(1970),233-254.

[4] R.C.Gonzalez, R.E.Woods and S.L.Eddins, digital image processing using MATLAB, New Jersey, Pearson Prentice Hall,(2004).

[5] G.T.Herman, On topology as applied to image analysis, computer vision, Graphics and Image processing, 52(1990),490-495.

[6] G.T.Herman and D.Webster, A topological proof of a surface tracking algorithm, computer vision, Graphics and Image processing, 23(1983),162-177.

[7] E.Khalimsky, R.Kopperman and P.R.Meyer, Computer graphics and connected topologies on finite ordered sets: Topology and Applications, 36(1990),1-17.

[8] V.Kovalevsky, "Finite topology as applied to image analysis", Computer Vision, Graphics and Image processing, 46(1989),141-161. 
[9] V.Kovalevsky, A new concept for digital geometry, In shape in picture, Springer, (1994),37-51.

[10] V.Kovalevsky, "Algorithms and data structures for computer topology", in G.Bertrand et al.(Eds), LNCS 2243, Springer, (2001) 37-58.

[11] V.Kovalevsky, Algorithms in digital geometry based on cellular topology In R.Klette. and J.Zunic(Eds.), LNCS 3322, Springer,(2004), 366-393.

[12] V.Kovalevsky, Axiomatic Digital Topology, Springer Mathematical image imaging and vision 26(2006), 41-58.

[13] V.Kovalevsky, Geometry of Locally Finite spaces, Publishing House Dr.Baerbel Kovalevski, Berlin, (2008).

[14] N.Levine, Semi-open sets and semi-continuity in topological spaces, American Mathematical Monthly, 70 (1963), 36-41.

[15] T.Pavlidis, Structural pattern Recognition, Springer, New York, (1977).

[16] T.Pavlidis, Algorithms for Graphics and Image processing, computer science press, Rockville, Maryland, (1982).

[17] G. Sai sundara Krishnan, N.Vijaya, Algorithm on tracing the boundary of medical image using abstract cellular complex, Machine vision and Image processing,International conference on,Taipei, IEEE-Xplore,(2012),141-144.

[18] Sang-Eon Han, Extension of continuity of maps between axiomatic locally finite spaces, International Journal of Computer Mathematics 88 (2011),2889-2900.

[19] N.Vijaya, G.Sai Sundara Krishnan, Characterization of semi-open subcomplexes in Abstract cellular Complexes, in proceedings of International conference on 'Computational Intelligence, cyber Security and Computational Models', Advances in intelligent systems and computing, Springer, 246(2014), 275-283. 
